\title{
Las universidades y el discurso ambientalista: las dos caras de la moneda $\stackrel{\perp}{ }$
}

\author{
Universities and the environmental discourse: two sides of the same coin \\ Les universités et le discours environnementaliste: les deux visages de la monnaie
}

Katherine Restrepo Quintero ${ }^{1}$, Contadora Pública; Juan D Cardona Hernández ${ }^{2 *}$, Contador Público; Hernán D Múnera Espinal ${ }^{3}$, Economista; Cielo C Zapata Marín ${ }^{4}$, Administradora de Empresas y Finanzas.

${ }^{1}$ Investigadora de la Facultad de Ciencias Empresariales, Corporación Universitaria Remington, Calle 51 No 51-27, Medellín, Colombia. Correo electrónico: katherinerq1989@gmail.com).

${ }^{2}$ Investigador de la Facultad de Ciencias Empresariales, Corporación Universitaria Remington, Calle 51 No 51-27, Medellín, Colombia. Correo electrónico: jcardona@uniremington.edu.co)

${ }^{3}$ Investigador de la Facultad de Ciencias Empresariales, Corporación Universitaria Remington, Calle 51 No 51-27, Medellín, Colombia. Correo electrónico: hernan.munera@uniremington.edu.co)

${ }^{4}$ Egresada de la Corporación Universitaria Remington, 2013. Asistente de investigación del trabajo: "La construcción del pensamiento ambiental en las universidades de la comuna 10 de Medellín”. Caso Universidad Autónoma Latinoamericana, Universidad Cooperativa de Colombia y Corporación Universitaria Remington. Teléfono y correo electrónico: 054-481-59-71 - 320-490-41-81; c.del.c.z.1971@hotmail.com

(Recibido: 20 de febrero de 2014; aceptado: 27 de mayo de 2014)

\section{Resumen}

La preocupación constante de las universidades e instituciones de educación superior por afrontar los retos de las nuevas formas de mercados y la cada vez más definida política en relación con la conservación del ambiente y el aprovechamiento de recursos, además de la búsqueda de mejorar las condiciones de vida y el desarrollo de estrategias de sostenibilidad ambiental, exigen que la reflexión, evaluación y el análisis en torno a los procesos, técnicas e instrumentos de la gestión ambiental se constituyan en una fase crucial para el entendimiento de los discursos empresariales con relación a la misma. En este sentido, se hizo relevante un análisis crítico e interdisciplinario de la manera cómo las

\footnotetext{
${ }^{\Perp}$ Para citar este artículo: Restrepo Quintero K, Cardona Hernández JD, Múnera Espinal HD, Zapata Marín CC. Las universidades y el discurso ambientalista: las dos caras de la moneda. RHS. Revista. Humanismo. Soc, Volumen 2 (1): 11-25.

${ }^{*}$ Autor para correspondencia: Juan David Cardona Hernández. Corporación Universitaria Remington. Calle 51 No 51-27, Edificio Remington. Facultad de Ciencias empresariales.E-mail: jcardona@remington.edu.co.
} 
universidades e instituciones de educación superior han estructurado sus discursos para sensibilizar a la comunidad universitaria a reducir el impacto negativo que generan al entorno; es así como el presente artículo, producto de investigación, indagó sobre los discursos ambientalistas usados desde lo administrativo, en la Universidad Autónoma Latinoamericana, la Universidad Cooperativa de Colombia y la Corporación Universitaria Remington, todas ellas con sede en la comuna 10 de la ciudad de Medellín. Se encontró que la legislación internacional y la nacional en relación con el ambiente, han guiado el actuar de las instituciones en la construcción de un pensamiento preocupado por el ambiente.

Palabras clave: ambiente, educación ambiental, gestión ambiental, pensamiento ambiental.

\section{Abstract}

The constant concern of universities and other higher education institutions to meet the challenges of new marketing alternatives, increasingly defined environmental policies and the use of resources, along with the search for better life conditions and sustainable strategies require reflection, evaluation and analysis of processes, techniques and instruments of environmental management to create a crucial phase for the understanding of corporate speech in these matters. In this sense, a critical and interdisciplinary analysis has become relevant regarding the way these institutions have structured their speech to sensitize their communities about the need for reducing their negative impact on the environment. This study asked about the environmentalist speech used by the administration of Universidad Autónoma Latinoamericana, Universidad Cooperativa de Colombia and Corporación Universitaria Remington, all three located in Comuna 10, Medellin. We found that international and national laws related with the environment have guided the actions of these institutions in the construction of environmental awareness.

Key words: environment, environmental awareness, environmental education, environmental management.

\section{Introducción}

"Produce una inmensa tristeza pensar que la naturaleza habla mientras el género humano no la escucha"

Victor Hugo

Las Instituciones de Educación Superior -IES-, entre ellas las universidades, han demostrado su viabilidad, reconocimiento, capacidad para transformarse y propiciar cambios sociales, debido al alcance y al ritmo de las exigencias del mercado en cuanto a las prácticas y los hábitos respecto del ambiente; por lo tanto, la sociedad tiende más a fundarse en comportamientos responsables con la conservación de los recursos no renovables desde propuestas sostenibles, comprometidas y reflexivas del papel, en las que se evidencian los aportes que cada área del saber está en capacidad de ofrecer para optimizar resultados. Las universidades y las IES procuran a través de la investigación desarrollar propuestas que tienen en cuenta la evolución cultural, socioeconómica y ecológicamente sostenible de los individuos, las comunidades y las naciones; por consiguiente, y dado que tienen que hacer frente a imponentes desafíos, la educación superior ha emprendido la transformación y la renovación que exigen los nuevos problemas sociales y las condiciones cambiantes del mercado, que determinan la conservación de recursos y la sostenibilidad de los proyectos.

Se buscó entonces, con el desarrollo del proyecto, caracterizar el pensamiento ambiental construido por algunas IES de la Comuna 10 de Medellín, caso Universidad Autónoma Latinoamericana, Universidad Cooperativa de Colombia y Corporación Universitaria Remington, que en términos del territorio que ocupan, se perciben como instituciones encargadas de las transformaciones sociales. También, con la intención de encontrar soluciones a estos desafíos y poner en marcha mecanismos para que los discursos se concreten en prácticas sustentables, desde el análisis de la información que cada institución aportó a la investigación.

Revista. Humanismo.Soc. 2014; Volumen 2: 11-25. 
En correspondencia con lo anterior, se planteó la siguiente hipótesis: "es posible identificar los principios del pensamiento ambiental que unifiquen el modelo normativo práctico y que permitan a las instituciones de educación superior la consolidación de un cuerpo coherente de estrategias que reivindiquen efectivamente, la optimización de recursos que comprometen el proyecto ambiental definido en los tratados internacionales, a que se suscribe Colombia”, puesto que ha sido desde las cumbres y las organizaciones multilaterales que se promueven propuestas alternativas que reivindican la naturaleza por encima del pensamiento instrumentalista del mercado.

\section{El ambiente ¿una problemática?}

La problemática ambiental a nivel global se ha constituido en uno de los factores determinantes en las políticas de desarrollo social en los países, tarea que según la Organización de Naciones Unidas debe ser liderada, a partir de la educación y la sensibilización de la sociedad, de las consecuencias que se derivan del descuido y abuso de los recursos naturales. La universidad como epicentro de las transformaciones sociales, implementa acciones en la sociedad a partir de sus tres funciones básicas: docencia, investigación y extensión, con la intención de proporcionar las bases del cambio en la sociedad por medio de propuestas alternativas, modelos, eventos, proyectos, entre otras herramientas; es decir, puede generar desde la aplicación de la teoría, una cultura en torno a la conservación del ambiente que permitan estudiar y analizar los distintos paradigmas que han nacido a la luz de la ciencia.

Pero, no se puede desconocer que involucra actores de tipo gubernamental, privado, ONG, instituciones educativas, entre otros; es decir, incluye a cada una de las partes que conforman la sociedad, puesto que se trata de un trabajo mancomunado, en el que se jalonan procesos de orientación y percepción del ambiente como parte inherente, sin el cual, la vida humana no sería posible. Es por esto que:

la construcción de una racionalidad ambiental es un proceso político y social que pasa por la confrontación y concertación de intereses opuestos, por la reorientación de tendencias (dinámica poblacional, racionalidad del crecimiento económico, patrones tecnológicos, prácticas de consumo: por la ruptura de obstáculos epistemológicos y barreras institucionales; por la creación de nuevas formas de organización productiva, la innovación de nuevos métodos de investigación, y la producción de nuevos conceptos y conocimientos). (Leff, 1994, pág. 20)

A pesar de lo anterior, el hombre en su afán de proveer de bienes y servicios para el consumo de la sociedad, ha dejado a un lado su preocupación por lo que le rodea, en este caso, la naturaleza; a través de la que consigue la materia prima para producir aquellos bienes que se hacen necesarios en la vida cotidiana del ciudadano común, pues éste tiene un concepto de calidad de vida, que lo lleva a inscribirse en una racionalidad instrumentalista donde "el ambiente es visto como un objeto... tiene que ser protegido y conservado frente a la invasión de elementos 'externos' que amenazan con deteriorar, contaminar o destruir." (Eschenhagen, 2007, pág. 88) Para lo que se proponen soluciones tecnológicas que no interrumpen la producción masiva; que hace que la responsabilidad del daño ambiental se convierta en un uso lógico de clasificación de residuos y tecnologías limpias, acciones que se quedan cortas frente a las catástrofes ambientales que se han hecho continuas y prolongadas en el tiempo.

Eschenhagen, explica también desde otros autores el ambiente como sistema, a partir de la teoría de sistemas que aborda el problema ambiental desde diferentes ángulos, político, social, cultural y económico; aquí se involucra desde el individuo hasta las instituciones que conforman la sociedad, puesto que la responsabilidad del deterioro es compartida. Por lo que el ambiente como sistema se entiende "como una relación que existe entre el ser humano y los ecosistemas" (Eschenhagen, 2007, pág. 89), la soluciones implementadas se hacen coercitivas a través de la imposición de leyes que regulan las actividades económicas, en las que quedan vacíos, lo que deja abierta la posibilidad a seguir con las prácticas poco sustentables ambientalmente; "es decir, se mantienen en cierta medida la racionalidad instrumental y economicista, aunque ampliando considerablemente el entorno y los factores a tener en cuenta" (Eschenhagen, 2007, pág. 90). Por último, parte de la complejidad y propone:

El ambiente como una crítica a la visión de mundo actual, porque se supone que existe una relación que se construye a través de un conocimiento que legitima y justifica una apropiación específica de una sociedad del entorno y que ahora crítica y pone en evidencia la insustentabilidad de la visión de mundo dominante 
actual. Por lo tanto se considera que "la crisis ambiental emerge como una manifestación de la exclusión de la naturaleza, la cultura y la subjetividad del núcleo duro de la racionalidad de la modernidad". (Eschenhagen, 2007, pág. 90)

Como se ha señalado, esa exclusión de la naturaleza se ha dado desde los discursos que direccionan la economía y política global, pues son estos, los que determinan los planes de gobierno de los presidentes de cada país, los cuales implican comportamientos en el mercado, que deja a la masa inmersa en ellos, sin pensar si son acordes o no con el ambiente. "El problema radica entonces en la imperceptibilidad del daño; el ser humano no es capaz de percibir, de visualizar, cómo su accionar diario contribuye a la contaminación y la sobreexplotación... pero la seguridad que le da la escasa contribución individual a la generación del problema impide que valore en su real dimensión su aporte al desastre ambiental. (Mercado, 2010, pág. 16)

Pero esa incapacidad de reconocimiento del impacto que se genera en el individuo, se ha vuelto la excusa más utilizada para responsabilizar individualmente de los niveles de contaminación; dejando a un lado la visualización real del problema ambiental, que compromete a cada persona y más aún a la empresas, puesto que estas producen a mayor escala y así mismo son los desechos generados, que en algunas ocasiones se presentan desastres que no son previstos por las compañías.

\section{Una mirada a los discursos que han propiciado el pensamiento ambiental en la educación superior}

El continuo llamado de la naturaleza frente a las acciones del hombre, refrenda la determinación del actuar de los seres humanos a una respuesta de los discursos económicos dominantes, pues éstos guían los imaginarios que se construyen frente al éxito, los niveles de consumo, la intervención de las empresas en el entorno natural. No ha sido gratuito empezar a ver la preocupación de la sociedad por proteger el ambiente, ya que las manifestaciones de desastres naturales han influenciado la conciencia del ser humano frente a las acciones y el lugar que le da a la tierra.

Es así como en 1972 las Naciones Unidas realizaron en Estocolmo, la Conferencia en torno al Medio Ambiente Humano, en la cual se abordaron las problemáticas ambientales que se han generado por el uso indiscriminado de los recursos naturales por parte de las empresas; este primer llamado de atención hizo que se pensará en la manera cómo se direccionaban las políticas de desarrollo económico de los países, por lo que se planteó un estilo de desarrollo más acorde con la naturaleza y el medio. Esto, implicó un cambio estructural en las prácticas empresariales y en los principios normativos a nivel económico, político y social; además, se consideró la educación como uno de los ejes fundamentales en el proceso de transformación social para disminuir el impacto ambiental.

Con el fin de darle continuidad al proceso de construcción de un pensamiento ambiental, se fundó en 1975 el Programa Internacional de Educación Ambiental -PNUMA- que se ha encargado en el caso de América Latina y el Caribe, de fomentar la educación ambiental a través de eventos académicos y la incursión en proyectos ambientales enmarcados desde la interdisciplinariedad; luego en Tbilisi (1977), se realizó la Conferencia Mundial sobre Educación Ambiental, a partir de la cual se propuso una formación que incluyera el cuidado del medio ambiente en la educación, que empezara desde la primaria hasta la universidad, para que niños, jóvenes y adultos se preocuparan por el futuro del planeta. En este mismo año, los gobiernos plantearon al PNUMA la intención de conformar una Red institucional en la que participaran universidades, Instituciones de Educación Superior, ONG'S, entre otros, en la formación ambiental de los países Latinoamericanos y que en 1982 se puso en marcha el Programa General de Ia Red de Formación Ambiental para América Latina y el Caribe, basado en la cooperación de los países de la región, con el apoyo catalítico del PNUMA (LEFF, 1996, pág. 105).

Después, se ratificaron los principios que se habían establecido en los anteriores eventos, en las conferencias celebradas primero en Moscú de 1987, en 1992 Producto del acuerdo alcanzado en Río de Janeiro en la conferencia de Naciones Unidas sobre el Medio Ambiente y el Desarrollo (Declaración de Río sobre el Medio Ambiente y el Desarrollo) y el plan de acción derivada de la misma, denominado Agenda 21; por lo tanto, las diversas instituciones sociales han procurado asumir prácticas y discursos en beneficio del proyecto de conservación y mejoramiento medioambiental en distintos ámbitos. De otro lado, las universidades e instituciones de educación, referentes y generadoras de 
modelos de acción, son quizás las llamadas de manera más urgente a presentar, por la vía de su propio accionar, ejemplos innovadores y efectivos que se ajusten a las necesidades identificadas para la optimización de recursos, manejo de residuos y espacios que vayan en beneficio de las condiciones de vida. Por consiguiente, en América Latina se empezaron a crear instituciones y grupos de interés alrededor del ambiente, en las que se han discutido a la luz de la teoría y de la interacción entre las ciencias sociales y naturales alternativas a la crisis ambiental; lo que generó diversas ramas temáticas en torno al ambiente y con un enfoque holístico, por ejemplo:

La filosofía ambiental, ha sido otro campo fértil del pensamiento ambiental latinoamericano, luego que fuera inaugurado por el uruguayo Daniel Vidart, quien en su refugio político en Colombia publicó en 1986 Filosofía Ambiental: epistemología, praxiología, didáctica. Este campo de reflexión filosófica anidó sobre todo en los Institutos de Estudios Ambientales (IDEA) que empezaron a establecerse en las universidades colombianas, luego del Primer Seminario sobre Universidad y Medio Ambiente, celebrado en Bogotá en 1985. Desde la creación del IDEA en la Universidad Nacional de Colombia, la construcción de un Pensamiento Ambiental ha sido una de sus tareas prioritarias, como respuesta al reduccionismo ecológico y tecnológico del estudio de los problemas ambientales y de sus soluciones. Esta fuente del pensamiento filosófico ambiental fue anidando en las universidades colombianas y extendiéndose hacia diferentes espacios de actuación a través de una Red de Nodos de Pensamiento Ambiental impulsado por un programa de formación liderado desde la sede Manizales de la Universidad Nacional de Colombia." (Leff E. , 2009, pág. 12)

Fue en el I Seminario Internacional Universidad y Medio Ambiente que inició un espacio para la discusión y argumentación de propuestas frente a la problemática ambiental que aqueja al planeta; se han discutieron acciones posibles desde la universidad Latinoamericana y Caribeña como lugares de construcción de conocimiento en torno al pensamiento ambiental, a la luz de los lineamientos planteados por el Programa Internacional de Educación Ambiental -PNUMA (1975), la Conferencia Mundial sobre Educación Ambiental (1977) y el Programa General de Ia Red de Formación Ambiental para América Latina y el Caribe (1982), las ponencias que se presentaron han evidenciado los procesos educativos en la incursión de nuevos cursos y asignaturas en materia Ambiental.
Este evento se constituyó como un hito en la historia del pensamiento ambiental.

En 1999 se realizó el segundo Seminario Universidad y Ambiente, liderado por la Universidad Autónoma de Occidente, en el cual se reflexionó sobre el impacto de la educación ambiental de cara a las necesidades del mercado, de un profesional más proactivo con el ambiente. De igual manera en el tercer seminario, que fue organizado por la Universidad de Ciencias Aplicadas y Ambientales en 2005, se evaluó la incorporación del ámbito ambiental en la educación en el área de formación de los pregrados y postgrados ofrecidos por las universidades, debido a que después de 20 años era necesario hacer un balance de la incursión de la educación en este aspecto.

Para el 2007 se celebró el IV Seminario Internacional Universidad y Ambiente, realizado por la Universidad de Ciencias Aplicadas y Ambientales con ayuda del Politécnico Grancolombiano, este evento giró en torno a la gestión ambiental institucional y ordenamiento de los campus universitarios, se puede mencionar entre las ponencias presentadas dos trabajos, uno, de autoría de Javier Benayas y David Alva titulado "la universidad como referente social del cambio hacia un futuro sostenible" (2007) retoman el papel que tiene la universidad en términos de la responsabilidad de preparar a las personas para afrontar los cambios que se dan con el paso del tiempo, pero, que la mirada del nuevo profesional tiene que ser consiente de los impactos que sus acciones generan al medio en el cual se desenvuelve, además de la importancia una cultura de respeto por la naturaleza, pues no se trata de ver los recursos naturales como parte de la dinámica empresarial de beneficio-riqueza sino de la supervivencia del hombre en el futuro por medio del uso racional de todos los recursos.

El otro trabajo que se encuentra en las memorias de este evento es "la gestión ambiental y el ordenamiento de campus universitarios: análisis y reflexiones a partir de algunas experiencias relevantes" (2007) presentado por Orlando Sáenz en el cual se realizó un llamado a las instituciones de educación superior a contar las experiencias de la implementación de sistemas de gestión ambiental en los campus, y es a partir de la gran acogida que tuvo la convocatoria de esta cuarta versión que se evidenció el avance que han tenido estas instituciones en la aplicación de principios y 
compromisos ambientales que van desde la academia, la investigación, la extensión hasta el nivel administrativo de la universidad. Éste escrito permite mirar y evaluar el proceso de aplicación de la norma ISO 14001 en estas instituciones.

En la quinta versión del Seminario Internacional Universidad y Ambiente liderado por la Universidad Tecnológica de Bolívar en el 2009, la temática propuesta fue "de cara al cambio climático" se analizó las prácticas que han llevado a esta crisis y cómo desde la universidad se pueden crear alternativas por medio de la investigación y la generación de conocimientos den respuestas y soluciones acertadas a la situación que vive la tierra. Es importante resaltar que aunque se reúnen varias organizaciones, no han logrado permear todas las Universidades e Instituciones de Educación Superior, pues de las 37 instituciones que son miembros de la Red Colombiana de Formación Ambiental, de Medellín sólo pertenecen la Universidad de Antioquia, la Universidad de Medellín, la Universidad Santo Tomás y la Universidad Cooperativa de Colombia, lo que hace que las otras instituciones apliquen sólo unas asignaturas dentro de las mayas curriculares de los programas académicos que ofrecen a la comunidad. Pero, las prácticas ambientales dentro de las mismas, se reducen al manejo de residuos y de algunos recursos como el agua; pero la sensibilización de estas instituciones debe llegar más allá de lo regulado, ya que se concibe como una manera de pensar y actuar de quienes la componen y rodean.

Este espacio se viene articulando desde las Redes Nacionales de Educación Ambiental y ha construido los Congresos Iberoamericanos de Educación Ambiental como rituales de reencuentro, reafirmación y proyección de los procesos educativos y formativos. Más allá de su trascendencia en el establecimiento de leyes, políticas y estrategias nacionales de educación ambiental, los actores de estos procesos han generado un verdadero movimiento social a favor de la educación ambiental, más allá de las instituciones y junto con las políticas públicas y los espacios de actuación en que se desarrollan. Lo que otorga su identidad a estos procesos $y$ a sus actores es el concepto de ambiente que funda el pensamiento ambiental latinoamericano. (Leff E. , 2009, pág. 13)

Otro referente, ha sido los Principios fundamentales correspondientes a los compromisos adquiridos por los países miembros de las Naciones Unidas en el mandato de la "Década de la Educación para el Desarrollo
Sostenible" 2005-2014, en el Primer Congreso Internacional de Educación Para el Desarrollo Sostenible: Una visión de la década; congreso que tuvo como objetivo conformar el Observatorio de Educación para el Desarrollo Sostenible para promover, consolidar y hacer seguimiento a la incorporación de los principios establecidos en la Década de Naciones Unidas de la Educación para el Desarrollo Sostenible 2005-2014. En el mismo sentido, la normatividad que cada institución se ha definido con la intención de acercarse al cumplimiento de estándares nacionales e internacionales para implementar prácticas responsables con la sociedad y el medio ambiente.

\section{Construcción del pensamiento ambiental en las universidades e Instituciones de Educación Superior}

"Desde que emerge la crisis ambiental a escala mundial, hacia principios de los años 70 , un grupo de intelectuales y académicos fueron atraídos por los vientos y mareas de esas nuevas ideas y propuestas, en torno a las cuales comenzó a darse un movimiento crítico y una respuesta desde América Latina." (Leff E. , 2009, pág. 4) En el intento por generar una conciencia ciudadana preocupada por el ambiente y destinada al cuidado y protección del mismo, espacios como los relacionados en el apartado anterior, ya que fueron éstos los que convocaron, socializaron y presentaron propuestas para que desde la educación se hiciera palpable una sensibilización del ser humano frente a las prácticas que afectan el ambiente.

De allí, varios docentes universitarios y pensadores de diversas profesiones como: Patricia Noguera, Augusto Ángel Maya, María Luisa Eschenhagen, todos estos han liderado el proceso de formación ambiental en Colombia; para el caso de América Latina y el Caribe, se hace esencial el trabajo realizado por el profesor Enrique Leff de la Universidad Autónoma de México, quien ha promovido a través de sus investigaciones y publicaciones la educación ambiental desde una visión real de las condiciones que tiene cada país, y no como una copia teorías que no se adecúan al contexto de cada región.

"La racionalidad ambiental seforja en la desconstrucción del pensamiento metafísico, científico y posmoderno; de la territorialización de la diversidad, la diferencia y la otredad; sobre la base de los potenciales ecológicos y de 
los saberes culturales que habitan los territorios del Sur. De allí nace y desde allí reivindicamos el pensamiento ambiental latinoamericano" (Leff E. , 2009, pág. 2). En "las universidades y la formación ambiental", artículo publicado en la revista Ciencias Humanas Leff hizo un recorrido por las conferencias, pactos e instituciones que han direccionado la formación ambiental en el ámbito internacional y regional para dar cuenta de la importancia de la educación en referencia al pensamiento ambiental, lo cual ha permitido la creación de programas desde pregrado hasta posgrado, sin dejar a un lado los niveles de formación básica y secundaría.

Las instituciones de educación superior como epicentro de las transformaciones sociales ha tomado la bandera en pro de la formación y sensibilización de la población civil en torno a las problemáticas ambientales y a las prácticas que desde el ámbito individual procuran la minimización o mitigación de los daños ambientales causados al entorno. Respecto de la formación del pensamiento ambiental en las universidades, éste pasa por diferentes etapas dentro del proceso de asimilación de forma consiente una postura colectiva sobre el ambiente, éstas se ubican en diferentes estadios donde se pueden identificar patrones de comportamiento que dan cuenta de posiciones que encuadran dentro de las tendencias que el tema ambiental vienen presentándose a nivel internacional.

En un primer estadio se identifica el potencial que los temas ambientales generan en la apertura de nuevos mercados verdes, jalonados por la proliferación de posturas, tratados y pronunciamientos a nivel de organizaciones no gubernamentales y de las conferencias que al respecto promueve las Naciones unidas y de los grupos ambientalistas que de alguna forma comienzan a generar, al menos publicidad en favor de los recursos y en contra de su destrucción.

En un segundo estadio de orden Nacional aparece la legislación como motor de los aspectos ambientales que obligan a las empresas al menos en el papel a considerar dentro de su quehacer diario lo ambiental todavía tratado como externalidad y que no genera compromisos como organización dentro de la empresa, sino apenas, como slogan de bienestar en favor de los recursos naturales. En este sentido el último fin de la inclusión de lo ambiental en los estatutos, reglamentos y demás aspectos normativos de la empresa se torna de conveniencia y no de conciencia.

La proliferación de los mensajes de protección, las restricciones impuestas desde los países Europeos y Estados Unidos a las importaciones a partir del endurecimiento de las reglas de tipo ambiental generaron en la empresa y la educación la necesidad de pensar el medio ambiente como alternativa de ganar mercados, podríamos llamarlo oferta verde de educación como proceso de mercadeo ante las nuevas condiciones mundiales.

\section{Estudio de caso}

\begin{abstract}
"Citando las palabras de Mario Díaz y recordando a Max Weber, la Universidad no debe dedicarse sólo a la formación de especialistas sin alma o, como lo manifestaba, de forma más cruda Estanislao Zuleta, a formar genios en su vida profesional, pero cretinos en la vida cotidiana; mostrando así, cómo la universidad tradicionalmente ha venido enfatizando sólo en la dimensión cognitiva olvidando las otras dimensiones del ser humano." (Rojas, 2008, pág. 13)
\end{abstract}

Se buscó sistematizar las experiencias institucionales universitarias en un contexto de alto impacto ambiental, como es la Comuna Diez, de la ciudad de Medellín; se escogieron instituciones, que por su razón de ser se inscriben en la posibilidad de hacer coherente su quehacer con el pensamiento ambiental propuesto por el Ministerio del Medio Ambiente, que a su vez se acoge a los pactos y acuerdos firmados a nivel mundial por Colombia. De esta manera es como se optó por estudiar las experiencias de la Universidad Cooperativa de Colombia -UCC-, Universidad Autónoma Latinoamericana -UNAULAy la Corporación Universitaria Remington -CUR-, ubicadas en el centro de Medellín. Estas instituciones se encuentran ubicadas en una zona de gran importancia para el mercado de bienes y servicios, además convoca a una población con unos intereses particularmente diversos, pues convergen personas de todos los estratos socioeconómicos de la cuidad. Por lo tanto las problemáticas que se evidencian en este territorio son focalizadas desde distintas disciplinas como la contabilidad, la sociología, la antropología, la administración y el derecho, pues la interrelación de las mismas generará una amplia gama de alternativas a los problemas que se viven en esta zona tan transitada 
diariamente y que tiene un alto número de estudiantes desde la educación primaria hasta universitaria.

Estas instituciones se asemejan, además de la ubicación, por el tipo estudiantes que se matriculan allí, pues éstos son parte del conglomerado de trabajadores que deben estudiar en horarios nocturnos y sabatinos; la infraestructura que poseen, son de edificios y casas de vieja data, que vinculan otros espacios cercanos en metros a la redonda. Por lo tanto no gozan en su mayoría de espacios verdes que permitan la visualización de una cultura en torno a la naturaleza.

Después de ser definidas las instituciones objeto de estudio, se empezó la primera fase del proyecto, se realizó un rastreo alrededor de los pactos y eventos a nivel internacional y nacional con la intención de contrastar cómo éstos han permeado de alguna manera el actuar de la educación superior. Allí se encontró que estos eventos son conocidos de forma general, pero la aplicación total en relación con los tratados ha sido lenta, pues es un trabajo mancomunado que no sólo desde se direcciona desde la normatividad impuesta por el Estado Colombiano, sino que hace que cada persona que compone la institución ya sea administrativo, estudiante, docente o viva en los alrededores del campus universitario debe estar permeado por ideas de cuidado y preservación del ambiente. Puesto que la universidad no es ajena a estas problemáticas y por lo tanto tiene bajo responsabilidad la socialización y generación de propuestas que permean a sociedad civil.

Para el análisis del aspecto ambiental en las universidades se tomó como punto de partida los aportes teóricos realizados por el profesor Enrique Leff en materia de educación ambiental; de manera específica para el caso Colombiano las experiencias publicadas por la profesora María Luisa Eschenhagen de la Universidad Pontificia Bolivariana (Medellín), la cual se ha dedicado al estudio de la problemática ambiental en la universidad. El grupo de investigación utilizó para el análisis del componente ambiental dentro de las instituciones objeto de estudio, el artículo "El tema ambiental y la educación ambiental en las universidades: algunos indicadores y reflexiones" en el que Eschenhagen planteó indicadores que a la luz del proyecto fueron útiles para descomponer la información obtenida en el rastreo bibliográfico desde la revisión del direccionamiento estratégico de estas tres instituciones, los indicadores aplicados fueron:
- Asignaturas ofrecidas en los pregrados.

- Oferta de posgrados.

- Medidas de gestión ambiental en las universidades.

En los apartados que siguen a continuación se hace el respectivo análisis de cada institución objeto de esta investigación.

\section{Universidad Cooperativa de Colombia sede Medellín}

La Universidad Cooperativa de Colombia se fundó en 1958 a partir de la iniciativa cooperativista en la que se buscaba fortalecer el sector solidario, por medio de la formación de personas, en razón de ese objetivo, ésta pasó por varios nombres hasta llegar al que actualmente se le conoce en el territorio nacional. Sólo hasta la década de los 90 abrió nuevas sedes, entre ellas, en la ciudad de Medellín; es aquí donde se concentró la investigación para evidenciar la construcción de un pensamiento en torno al ambiente.

El primer análisis realizado fue la revisión de la visión, misión, principios y valores que han constituido la razón de ser de esta institución, allí se encontró que en la visión, misión y valores no hay mención del aspecto ambiental; pero en los principios se hace el siguiente reconocimiento:

La Universidad cree y practica la Responsabilidad Social Universitaria, asumiendo un compromiso institucional con el cuidado y promoción de ambientes naturales y sociales sanos y justos, con un ejercicio profesional y académico ético y responsable con los otros presentes y futuros y con una convicción de que un mundo mejor e incluyente se construye con la participación de todos (Universidad Cooperativa de Colombia, 2013).

Aunque, no se haya reconocido de manera inmediata en los elementos analizados inicialmente, se evidenció la constante preocupación que ha tenido la Universidad Cooperativa de Colombia por abordar e implementar estrategias en torno a las problemáticas ambientales; el cual se ha dado desde el estudio minucioso de los eventos académicos, las cumbres y conferencias nacionales e internacionales, que han girado en torno al ambiental. Éstas tomaron cuerpo y vida por medio de la normatividad creada por el órgano legislativo y ejecutivo de Colombia, debido a la suscripción a pactos asumen compromisos, para abanderar los desafíos y llamados de la educación actual. 
Es importante tener en cuenta que el auge de la responsabilidad social empresarial y la gestión ambiental, se han convertido en iconos de empresas modelo, puesto que la sociedad tiene en cuenta la imagen y los comentarios alrededor del objeto social que desarrollan dichas compañías. Las universidades e Instituciones de educación superior no son ajenas a esta situación, debido a que su razón de ser es la formación de profesionales, que direccionarán y harán frente a los retos que día a día impone el mercado. Es así como la Cooperativa, empezó a inscribirse en la cultura de la gestión ambiental; para el año 2008 con la redacción de los "Documentos de Integración para el Desarrollo No. 12" realizada por Luis Francisco Rojas de la Dirección de Planeación Nacional de la Universidad. En este documento quedaron plasmados los lineamientos teóricos y normativos que guían la gestión ambiental para la institución, lo que determinó la firma del

Acuerdo del Consejo Superior 07 de mayo de 2008 como mecanismo de concreción de la responsabilidad social universitaria en relación con el desarrollo sostenible en el ámbito global, nacional, regional y local. El SGA refuerza los valores, recursos y bienes colectivos, articulando la dimensión ambiental en sus funciones misionales como la docencia, la investigación, la proyección social y la gestión, las cuales, analizadas conjuntamente con políticas nacionales y locales, se constituyen en referente para alcanzar los objetivos del desarrollo sustentable. (Universidad Cooperativa de Colombia, 2013)

Ha sido desde la aplicación del Sistema de Gestión Medioambiental-SGMA-ISO 14001, quelauniversidad ha proporcionado prácticas más sustentables con el ambiente; ésta cuenta con un departamento de Gestión Ambiental que es el encargado de prevenir, mitigar y resolver todos los impactos que se generan por el desarrollo del objeto social, en este caso el servicio de educación superior, el cual implica una sensibilización de toda la comunidad inmersa en la institución. En cuanto a las basuras, han dispuesto las canecas de colores (azul, gris y verde) para que la comunidad universitaria: estudiantes, administrativos, docentes y personal externo realice la separación de residuos de acuerdo con las respectivas especificaciones que da la norma y que se encuentran en la parte superior de cada caneca.

En los baños se encuentran equipados con sensores para que la luz se encienda sólo en el momento en que ingresa una persona, tienen además sistema para el control del uso del agua en los lavamanos, puesto que las perillas convencionales pueden permitir derrames de aguas limpias. Además, cuentan con campañas en pro del cuidado del medio ambiente con el fin de crear una cultura que garantice unas prácticas amigables con éste, que son aplicadas a cada una de las sedes;

En la actualidad la Universidad adelanta un mega proceso encabezado a la cabeza de la Dirección Nacional de Gestión Documental, su propuesta principal es poner en marcha un proyecto llamado proyecto "OFICINA SIN PAPEL, UNIVERSIDAD CON MEMORIA". Su objetivo general es establecer e implementar una política cero papel, que permita remplazar y reducir el flujo documental de papel por soportes electrónicos, mediante el fortalecimiento de nuevos mecanismo de comunicación y desarrollando estrategias que disminuyan los costos y tiempos administrativos de los diferentes procesos internos, proporcionando las herramientas y los insumos necesarios para que la comunidad de la institución genere una disminución del uso del papel. (Universidad Cooperativa de Colombia, 2013)

Para llevar a cabo el proyecto anterior, implementarán la estrategia de "ADOPTA UNA PLANTA", en éste se procura que las personas se vinculen con sembrar, cuidar y sostener una planta, ya que estos seres vivos se encargan de proveer de oxígeno a través del proceso de fotosíntesis, sin dejar a un lado la tranquilidad que generan en espacios fríos como son las oficinas, puesto que son lugares parcos. También, tienen proyectado para un periodo de cuatro años aproximadamente la construcción de la nueva sede de la Universidad Cooperativa de Colombia, que tiene un valor de $\$ 35.000$ millones de pesos, éste es uno de los más ambiciosos, porque intenta transformar la ciudad de Medellín con un Universidad que los edificios a construir son sostenibles con el ambiente. El proyecto se empezará a ejecutar en enero de 2014, tendrá tres etapas puesto que contará con un complejo académico que se complementara con espacios para la cultura, el deporte y la educación. Estará ubicado en el actual Parque Córdoba. En cuanto al tema ambiental la universidad se ha dicho que:

La primera etapa de la nueva infraestructura física de la UCC contará con un sistema de ventilación natural, con orientación norte - sur de acuerdo con el régimen de vientos de la ciudad; también tendrá un importante sistema de iluminación natural, que disminuye sustancialmente el consumo de energía 
eléctrica y permite que se requerirá un sistema de aire acondicionado de bajo consumo en espacios puntuales de la edificación. Tendrá aprovechamiento de aguas lluvias para aparatos sanitarios y sistemas de aseo, así como uso de materiales de construcción de fácil y bajo costo de mantenimiento, haciendo de este, un proyecto respetuoso con el ambiente y muy seguro para toda la comunidad universitaria.

Por otra parte, con respecto a los indicadores: asignaturas ambientales en los programas de pregrado y los programas de posgrados que ofrece la universidad; lo cuales componen el aspecto de la educación ambiental como parte esencial de la construcción del pensamiento ambiental en la formación de profesionales integrales y conscientes de la problemática ambiental. La universidad cuenta en esta sede con los programas: Administración de empresas, Contaduría Pública, Comunicación Social, Derecho, Ingeniería Civil, Licenciatura en Matemáticas e Informática, Licenciatura en Tecnología e Informática; los pensum de éstos no fueron proporcionados, por lo cual este indicador no fue evaluado.

En lo que respecta a la oferta de posgrados académicos cuentan con cinco especializaciones, de las cuales tres de ellas son de las ciencias de la salud y las restantes hacen parte del área de la educación; con relación a las maestrías en esta sede se cuenta con dos una en Gestión de Organizaciones y otra en Psicología de la Educación. Por lo tanto este indicador no aplica para esta institución.

\section{Universidad Autónoma Latinoamericana}

En la misión y visión institucional no aparece de manera explícita alguna mención a lo ambiental. De manera implícita, aunque habría que indagar más en el asunto, se podría pensar en que la mención que se hace a la contribución al desarrollo en el contexto nacional e internacional en la misión lleve de por sí lo ambiental. Este aspecto implícito podría estar también en la visión, aunque es curioso que mencionen diferentes ámbitos de la vida social y se deje de lado lo ambiental. Por otra parte, ni en los estatutos y reglamentos aparece de manera explícita lo ambiental, o es tratado en contextos en que se entiende más hacia lo que es la convivencia. Por ejemplo en los estatutos, reformados en 2004, cuando se habla de ambiente (palabra utilizada sólo una vez en el texto) se hace en el contexto de los objetivos de la institución en el literal f como sigue:
F) Propiciar en los alumnos un espíritu reflexivo y un conocimiento racional orientados al logro de la autonomía personal, en ámbito de libertad de pensamiento y de pluralismo ideológico que tenga en cuenta la universalidad de los saberes y la particularidad de las formas culturales existentes en el país, por lo que reinará un ambiente de libertad, especialmente de enseñanza, de aprendizaje, de investigación y de cátedra (Estatutos UNAULA, p. 13).

De igual forma, en el reglamento académico de la institución no se hace mención a la palabra ambiente, pero sí se menciona la palabra naturaleza en su artículo 7, éste reza:

Artículo $7^{\circ}$. El plan de estudios es el conjunto de las materias básicas, profesionales electivas y de énfasis distribuidas por niveles, fijado por las autoridades universitarias, con el objeto de capacitar al estudiante para ser un profesional eficiente, imaginativo, creativo, solidario, tolerante, investigador, democrático y defensor de los derechos humanos y de la naturaleza (Reglamento académico, p. 14-15).

En el reglamento interno de trabajo se hace mención a lo ambiental sólo dos veces. La primera vez se menciona en el Capítulo XVIII "Mecanismos de prevención del acoso laboral y procedimiento interno de solución", en el artículo 50 se dice:

Artículo 50. Los mecanismos de prevención de las conductas de acoso laboral previstos por la Universidad, constituyen actividades tendientes a generar una conciencia colectiva conviviente, que promueva el trabajo en condiciones dignas y justas, con armonía entre quienes comparten la vida laboral empresarial y el buen ambiente en la Universidad y proteja la intimidad, la honra, la salud mental y la libertad de las personas en el trabajo (Reglamento interno de trabajo, p. 29).

A su vez, artículo 52 del mismo capítulo en el numeral 3 literal a) se plantea "Evaluar continuamente la vida laboral de la Universidad en relación con el buen ambiente y la armonía en las relaciones de trabajo, tomando en consideración las sugerencias que se estimen necesarias" (Reglamento interno de trabajo, p. $31)$.

Cuando en dicho reglamento se menciona la palabra naturaleza, se hace en relación a aspectos legales. Por ejemplo cuando se habla de un procedimiento interno se menciona que este "pretende desarrollar las características de confidencialidad, efectividad y 
naturaleza conciliatoria señaladas por la ley para este procedimiento" (Reglamento interno de trabajo, p. 30). La palabra naturaleza está signada más en referencia a una situación relacional que a la naturaleza entendida como la fauna o flora o su acepción ecológica. Esto mismo se puede evidenciar en el artículo 51 numeral 2 cuando se habla de los mecanismos de prevención de acoso laboral y se plantea que hay "Espacios para el diálogo, círculos de participación o grupos de similar naturaleza para la evaluación periódica de vida laboral" (Reglamento interno de trabajo, p. 29).

En el Reglamento de Bienestar Universitario la palabra ambiente se menciona una vez en el capítulo 1 artículo 16 en el siguiente sentido: "El área lúdico deportiva realizará actividades que motiven y desarrollen la actividad física y mental de la comunidad universitaria, generando espacios de ambiente saludable que propicien la formación integral" (Reglamento Bienestar Universitario, p. 9). Por otra parte, la palabra naturaleza es mencionada 4 veces para indicar que ciertos espacios o actividades son de "naturaleza voluntaria" o para definir la "naturaleza" del departamento de Bienestar Universitario.
Adicional a lo anterior es significativo destacar que en octubre de 2012 la institución realizó en su Cátedra Universitaria en Derecho Penal "Guillermo Duque Ruiz" presentó una conferencia que giró sobre el tema "Delios contra recursos naturales y medio ambiente". Lo cual muestra que desde la institución se ha empezado a discutir el tema de lo ambiental. Por último cabe resaltar que en ninguno de los documentos revisados se utiliza la palabra medio ambiente, o recursos ambientales.

Ahora, en lo que respecta a la gestión ambiental, no existe un departamento que se encargue o responsabilice del direccionamiento estratégico en torno a prácticas amigables con el ambiente; cuentan con canecas para la clasificación y depósito de las basuras, además de la respectiva indicación de cómo realizarlo. Con relación al consumo de agua y energía no se encontró en los baños dispositivos que propendan por el ahorro de estos recursos, debido a que las instalaciones no han sido adecuadas y remodeladas para fines ambientales. De otro lado, el indicador de asignaturas ambientales ofertadas por pregrado con que cuenta la Universidad Autónoma Latinoamericana se relaciona en la tabla 1.

Tabla 1. Asignaturas ambientales ofrecidas en los pregrados UNAULA.

\begin{tabular}{lll} 
Programa & Asignatura & Semestre \\
Administración de Empresas & Gestión ambiental & Sexto \\
Contaduría Pública & Contabilidad social y ambiental & Octavo \\
Economía & Economía ambiental I & Noveno \\
& Economía ambiental II & Décimo \\
Licenciatura en Ciencias Sociales & Medio Ambiente & Octavo \\
Ingeniería Industrial & No aplica & \\
Ingeniería Informática & No aplica & \\
Derecho & No aplica & \\
\hline
\end{tabular}

*Información encontrada en la página web de la institución.

En total la institución ofrece siete programas de pregrado, de éstos sólo en 4 programas se ofrecen cursos en los que se hace referencia a lo ambiental. Se destaca que el programa de economía que tiene dos cursos sobre lo ambiental, éste es el que más se incluye lo ambiental. De otro lado, en torno a los posgrados ofrecidos, tiene un total de 17 especializaciones y 1 maestría en
Educación y derechos humanos que no incluye curso sobre el ambiente, frente a las especializaciones, sólo en la especialización en Gerencia Estratégica de Costos y en Revisoría Fiscal, se dictan respectivamente en el orden en mención la asignatura costos ambientales y control ambiental que pertenecen al área contable. 


\section{Corporación Universitaria Remington}

Ingresó en el proceso de construcción de su pensamiento ambiental, desde la mención de este componente dentro del proceso de formación de profesionales en los documentos institucionales: misión, visión y valores corporativos. En la misión deja plasmada "la formación integral de la persona con competencias profesionales, visión global y valores éticos, morales, políticos, económicos, ambientales y culturales" (CUR, 2013); en la visión se percibe como un compromiso social e internacional en torno al medio ambiente. Por último, dentro de los valores se plantea la valoración del espacio: explicado de la siguiente forma:

"Es el valor representado en el cuidado, recuperación y sostenimiento del ecosistema, representado este desde el microsistema propio de cada individuo. Desprendido de lo anterior el desarrollo sostenible como preocupación actual de la humanidad, debe convocar a la reflexión y a la acción académica que propenda por actuaciones humanas que tengan que ver, entre otras con: combatir el cambio climático; hacer uso más responsable de los recursos energéticos y frenar en la medida de lo posible desgaste terrestre" (CUR, 2013).

En referencia al direccionamiento estratégico de la CUR se evidencia un esfuerzo en insertar lo ambiental dentro de sus políticas y principios institucionales, esto se ve reflejado en los procesos misionales de la institución, la docencia, la investigación y la extensión. Se han implementado las canecas para la clasificación de los residuos, además del uso de sensores para encender la luz de los baños, perillas del agua que tienen un límite de tiempo para el paso de ésta y sensores para los secadores eléctricos de los baños, con la intención de no usar más papel del que se necesita; allí también se tienen frases alusivas a la protección de los recursos naturales, que invitan a un comportamiento que no afecte al ambiente y que se envían a los correo electrónicos de la comunidad universitaria y se publican en "Remington al día", esto ha determinado la iniciativa del uso de papeleras en cartón para el reciclaje de papel de las oficinas.

De igual manera, el proceso de inserción de lo ambiental en el direccionamiento estratégico de la Corporación se muestra de una forma explícita en la inclusión del tema ambiental en el currículo de los diferentes programas, como elemento transversal; en este sentido se sirven asignaturas que discuten el problema ambiental desde las diferentes ciencias que convergen en los programas de formación profesional con que cuenta la institución; en la tabla 2, se puede evidenciar esto:

Tabla 2. Asignaturas ambientales ofrecidas en los pregrados CUR.

$\begin{array}{llc}\text { Programa } & \text { Asignatura } & \text { Semestre } \\ \text { Derecho } & \text { Ecología Humana y Ambiental } & 10 \\ \text { Medicina } & \text { No aplica } & 1 \\ \text { Veterinaria } & \text { Ecología - Medio Ambiente } & 4 \\ \text { Ingeniería de Sistemas } & \text { Ecología Humana y Ambiental } & 3 \\ \text { Contaduría Pública } & \text { Ecología Humana y Ambiental } & 9 \\ & \text { Control en la Gestión Ambiental } & 3 \\ \text { Administración de Empresas } & \text { Ecología Humana y Ambiental } & 2 \\ \text { y Finanzas } & & \\ \text { Enfermería } & \text { Salud y Medio Ambiente } & 2 \\ \text { Arquitectura } & \text { Ecología } & 1 \\ & \text { Paisaje y Ecosistema } & 3 \\ & \text { Ciudad y Territorio } & 9 \\ \text { Siseño Visual } & \text { Sostenibilidad y Paisaje } & 10 \\ & \text { Ecología } & 1 \\ & \text { Paisaje y Ecosistema } & 3\end{array}$

Revista. Humanismo.Soc. 2014; Volumen 2: 11-25. 
En total la CUR ofrece a la sociedad nueve programas de pregrado, del cual sólo uno, no tiene dentro de su oferta académica asignaturas en torno al ambiente; la materia "Ecología Humana y Ambiental" es transversal para cinco programas profesionales, las otras cuentan con asignaturas propias del área de conocimiento que guía el proceso de formación. La institución cuenta con cinco especializaciones, de las cuales tres son de las ciencias jurídicas, una del área de informática y la otra tiene que ver con las ciencias económicas. Se tiene el proyecto de la presentación de una especialización en gestión ambiental y hábitat ante el Ministerio de Educación.

\section{Conclusiones}

A lo sumo, las memorias de encuentros y seminarios han posibilitado el intercambio de ideas y la construcción de instrumentos conjuntos y que pueden consultarse en las páginas web institucionales, lo que ha permitido que el conocimiento sobre esta área del saber sea socializado y deje memoria para aquellos que apenas se interesan por el tema. También, es una carta de navegación para evaluar los planes de acción institucionales que han permitido alcanzar los objetivos propuestos por los gobiernos y las ONG.

La importancia de los eventos no sólo radica en el control que la sociedad hace de los compromisos adquiridos en estos, sino que es la reafirmación de una causa y un proyecto que se hace más ambicioso a la hora de implementar acciones que mitiguen, aproveche, sensibilice y promueva una cultura que se preocupe por el ambiente, no como recursos para la producción de bienes y servicios, pero si como el elemento más importante del que depende la vida en la tierra. Estas ideas de conservación de la naturaleza, se ha convertido en el discurso que guía el actuar de instituciones, empresas, gobiernos, ONG, entre otras organizaciones y ha hecho que la sociedad se involucre a aportar ideas que cuentan con todas las personas que la componen.

El trabajo realizado por el profesor Enrique Leff se constituye en una reconstrucción del pensamiento ambiental latinoamericano, que recoge las verdaderas raíces para repensar lo que significa el ambiente en países con tanta abundancia de recursos verdes, que se hacen atractivos para la inversión extranjera y explotación de recursos naturales sin ningún cuidado por el bien común. Es importante, mencionar que para llegar a entender el problema ambiental como una consecuencia del pensamiento cultural y de la diversidad que éste genera en la sociedad, se ha hecho necesario las convergencias de las ciencias naturales, sociales, humanas y económicas, lo que deja atrás las rencillas entre las ciencias puras y sociales.

De otro lado, la construcción del pensamiento ambiental en las universidades se ha direccionado desde la normativa que genera el Ministerio de Educación, el cual hace hincapié en una formación integral y consciente de las acciones de los profesionales, los cuales han pasado por un proceso de formación que integra las diferentes ciencias, por lo que las asignaturas transversales se hacen productivas y prácticas en el ejercicio profesional. Al igual, la gestión ambiental abarca aspectos administrativos, educativos y de transformación del hombre, puesto que permea y organiza el actuar de éste en relación con los discursos sustentables en torno al ambiente.

Además, se evidenció que la inclusión de las asignaturas dictadas en los programas de pregrado, dan cumplimiento de la ley 115 de 1994 por la cual se expide la Ley General de Educación, que en el artículo $5^{\circ}$ Fines de la Educación. De conformidad con el artículo 67 de la Constitución Política, en el que se especifica en el literal que la educación tendrá como uno de sus fines, "la adquisición de una conciencia para la conservación, protección y mejoramiento del medio ambiente, de la calidad de la vida, del uso racional de los recursos naturales, de la prevención de desastres, dentro de una cultura ecológica y del riesgo y de la defensa del patrimonio cultural de la nación". Y en concordancia con la Política Nacional de Educación Ambiental de 2002. 


\section{Referencias}

Benayas, Javier, y Alba, David (2007). La Universidad como referente social del cambio hacia un futuro sostenible. Universidad Autónoma de Madrid. Vicerrectorado de Campus y Calidad Ambiental. Memorias de Ponencias del IV Seminario Internacional Universidad y Ambiente. Bogotá.

Corporación Universitaria Remington (2013). Información institucional. Disponible en Internet: http://corporacion. remington.edu.co/corporativo/mision-vision-valores Consultado 20.03.13

Eschenhagen, María (2007). La educación ambiental superior en América Latina: una evaluación de la oferta de posgrados ambientales. En: THEOMAI. Estudios sobre sociedad y desarrollo, p. 87-107

Granada, L F. (2006). Gestión ambiental, filosofías, conceptos, instrumentos y herramienta Cali: Editorial Universidad Libre Seccional Cali.

Ideam (s.f). Lineamientos para una política ambiental urbana en Colombia.Disponible en Internet: http://www.ideam.gov.co/ apc-aa/img_upload/467567db4678d7b4443628f8bc215f32d/ POLITICA_URBANA.pdf(13 oct. 2008). Consultado 08.08.12

ISO 14001:2004. Sistemas de gestión ambiental.

ISO 14004:2004. Sistemas de gestión ambiental. Directrices generales sobre principios, sistemas y técnicas de apoyo.

Leff, Enrique (1994). Ciencias sociales y formación ambiental. Barcelona: Editorial Gedisa, 321p.

Leff, Enrique (1996).Convidados especiais: Las universidades y la formación. En Revista de Ciencias Humana, 14 (20), p.103-124

Leff, Enrique et al (2003). La complejidad ambiental. 2. Ed. Argentina: Siglo XXI, 314 p

Leff, Enrique (2004.). Racionalidad ambiental la reapropiación social de la naturaleza. México: Siglo XXI Editores 509 p.

Leff, Enrique. (2009) Pensamiento ambiental latinoamericano: Patrimonio de un saber para la sustentabilidad. En ISSE Publicación Ocasional, p. 1-15

Leff, Enrique (2010). Ecología y capital: racionalidad ambiental, democracia participativa y desarrollo sustentable. 2. ed. México: Siglo XXI Editores. 437 p.
Lef Enrique (2011). Sustentabilidad y racionalidad ambiental: hacia "otro" programa de sociología ambiental. En: Revista Mexicana de Sociología (México). Vol. 73, No. 01, Ene.-Mar. 2011. p. 5-46.

Mercado, Oscar. (2010). Universidad y medio ambiente. En: Triología. Ciencia - Tecnología -Sociedad, p. 15-23.

Montes, Ángela, y colaboradores. (1998) La gestión Ambiental en las organizaciones Gubernamentales y no gubernamentales en Manizales y su área metropolitana. Revista científica Lunazul. Universidad de Caldas. Disponible en Internet:

ht t p: // lunazul.ucaldas.edu.co/ downloads/223098aaRevista9_10_10.pdf Consultado 15.08.12

Ospina, Samuel. (2004). Gestión Ambiental Local. Primera Edición. Universidad Tecnológica de Pereira.

Rojas, Luis. (2008). Sistema de Gestión Ambiental. Bogotá: Universidad Cooperativa de Colombia.

Sáenz, Orlando (2007). Gestión ambiental y ordenamiento de campus universitarios. Análisis y reflexiones a partir de algunas experiencias relevantes. Primer curso internacional universidad y ambiente. Gestión ambiental institucional, ordenamiento de campus universitarios, UDCA, UDUAL. Bogotá.

Serrano, José (1997). "Principios filosóficos de la Gestión Ambiental”. En: Ballesteros, Jesús y Pérez, José (Compiladores). Sociedad y Medio Ambiente. Madrid: Editorial Trotta.

Tobasura, Isaías (1998) La investigación acción participativa frente a la crisis ambiental. Revista científica Lunazul. Universidad de Caldas. Disponible en Internet:

ht t p : / / lunazul. ucaldas.edu.co/ downloa ${ }^{a}$ s/8a15d92aRevista9_10_7.pdf Consultado 07.12.13

Udual. Declaración de la Universidad Latinoamericana en el siglo XXI. Unión de Universidades de América Latina. México. Disponible en Internet: http://www.udual.org. Consultado 03.08.12

Universidad Cooperativa de Colombia. Página web institucional. Disponible en Internet: http://www.ucc.edu.co Consultado 15.03.13

Unaula. Página web institucional. Disponible en Internet: http://www.unaula.edu.co/ Consultado 04.04.13 
Universidad Autónoma Latinoamericana. Estatutos y reglamentos. Disponible en Internet: http://www.unaula.edu. co/universidad/estatutos Consultado 04.04.13
Von Aecken, Berta (2001). Institucionalización del tema ambiental en la Universidad Javeriana. Bogotá: Pontificia Universidad Javeriana. 